Pacific Journal of Mathematics

PEAK INTERPOLATION SETS FOR SOME ALGEBRAS O 


\title{
PEAK INTERPOLATION SETS FOR SOME ALGEBRAS OF ANALYTIC FUNCTIONS
}

\author{
A. M. Davie AND B. K. ØKsendal
}

\begin{abstract}
For certain algebras of analytic functions on holomorphically convex sets in $C^{n}$ metric sufficient conditions are given for a set (not necessarily compact) to be an interpolation set. The results extend the Rudin-Carleson theorem for the disc algebra.
\end{abstract}

Let $K$ be a compact subset of $C^{n}$ which is holomorphically convex, i.e. $K$ is the intersection of a decreasing sequence of pseudoconvex domains (see [4], Ch. 2). We denote by $H(K)$ the uniform closure on $K$ of the algebra of all functions analytic in a neighborhood of $K$, and by $A(K)$ the algebra of all continuous functions on $K$ analytic on $K^{0}$ (the interior of $K$ ). If $E$ is any subset of the boundary $\partial K$ of $K$ then we denote by $H_{E}^{\infty}$ the algebra of all bounded continuous functions on $K^{0} \cup E$ which are analytic on $K^{0}$. We show that if the boundary of $K$ is well behaved at each point of $E$, and $E$ satisfies a metric condition which says roughly that $E$ has zero 2-dimensional measure in the directions of the complex tangent and zero one dimensional measure in the orthogonal direction, then $E$ is a peak interpolation set (in an appropriate sense) for $H_{E \cup(\partial K \backslash \bar{E})}^{\infty}$. If $E$ is compact then it is a peak interpolation set in the usual sense ([2], p. 59) for the uniform algebra $H(K)$. We show also that if $E$ has zero one-dimensional measure then the conditions on $\partial K$ can be relaxed.

We say that $\partial K$ is strictly pseudoconvex in a neighborhood of a point $\zeta \in \partial K$ if there is an open neighborhood $V$ of $\zeta$ such that $V \cap$ $\partial K$ is a $C^{2}$-submanifold of $V$ and the Levi form is positive definite at $\zeta$. Then we can find an open neighborhood $V$ of $\zeta$ and a $C^{2}$ strictly plurisubharmonic function $\rho$ in $V$ such that $K \cap V=\{z \in V: \rho(z) \leqq 0\}$ and $\operatorname{grad} \rho \neq 0$ on $V \cap \partial K$. (See [3] Prop. IX. A4).

Lemma 1. Let $K$ be a holomorphically convex compact set in $C^{n}$ and let $\zeta$ be a point of $\partial K$ in a neighborhood of which $\partial K$ is strictly pseudoconvex. We can find positive numbers $m_{\zeta}$ and $M_{\zeta}$ and $G_{\zeta} \in H(K)$, such that

(a) $\operatorname{Re} G_{\zeta}(z) \geqq m_{\zeta}|\zeta-z|^{2}, z \in K$

(b) $\operatorname{Re} G_{\zeta}(z) \leqq M_{\zeta}|\zeta-z|^{2}, z \in \partial K$

(c) $\operatorname{grad}\left(\operatorname{Re} G_{\zeta}\right)(\zeta)=-\operatorname{grad} \rho(\zeta)$.

Proof. Put 


$$
F(z)=\sum_{i=1}^{n} \frac{\partial \rho(\zeta)}{\partial \zeta_{i}}\left(z_{i}-\zeta_{i}\right)+\frac{1}{2} \sum_{i, j=1}^{n} \frac{\partial^{2} \rho(\zeta)}{\partial \zeta_{i} \partial \zeta_{j}}\left(z_{i}-\zeta_{i}\right)\left(z_{j}-\zeta_{j}\right)
$$

Then the Taylor expansion of $\rho$ about $\zeta$ is

$$
\rho(z)=2 \operatorname{Re} F(z)+\sum_{i, j=1}^{n} \frac{\partial^{2} \rho(\zeta)}{\partial \zeta_{i} \overline{\partial \zeta_{j}}}\left(z_{i}-\zeta_{i}\right)\left(z_{j}-\zeta_{j}\right)+o\left(|z-\zeta|^{2}\right) .
$$

Since $\rho$ is strictly plurisubharmonic at $\zeta$ it follows that, shrinking $V$ if necessary, we can find $m>0$ with $\operatorname{Re} F(z) \leqq-m|\zeta-z|^{2}$ for $z \in K \cap V$. Since $\rho=0$ on $\partial K \cap V$ we also deduce that for some constant $M$

$$
\operatorname{Re} F(z) \geqq-M|\zeta-z|^{2} ; z \in \partial K \cap V \text {. }
$$

Choose a pseudoconvex open neighborhood $U$ of $K$ so that $\operatorname{Re} F<0$ on an open neighborhood $W$ of $\partial V \cap U$ in $U$. Let $W_{1}=W \cup(V \cap U)$ and $W_{2}=W \cup(U \backslash \bar{V})$, so that $W_{1} \cup W_{2}=U$ and $W_{1} \cap W_{2}=W$. By solving a Cousin problem in $U$ (see [4], Theorem 5.5.1) we can find analytic functions $g_{1}$ and $g_{2}$ on $W_{1}$ and $W_{2}$ respectively such that $g_{2}-g_{1}=F^{-2} \log F$ on $W$.

$$
\text { Put } h(z)= \begin{cases}F(z) \exp \left(F(z)^{2} g_{1}(z)\right), & z \in W_{1} \\ \exp \left(F(z)^{2} g_{2}(z)\right), & z \in W_{2}\end{cases}
$$

The definitions agree on $W$ so $h$ is an analytic function on $U, h(z)=0$ only when $z=\zeta$, and in a neighborhood of $\zeta, h(z)=F(z)+0\left(|z-\zeta|^{3}\right)$. Thus $\operatorname{Re} h \leqq 0$ near $\zeta$, so there exists $\varepsilon>0$ such that if $z \in K$ and $|h(z)-\varepsilon| \leqq \varepsilon$ then $z=\zeta$. Put

$$
G(z)=-\frac{h(z)}{\varepsilon-h(z)}, z \in K .
$$

Then $G \in \bar{H}(K), \operatorname{Re} G(z)>0$ for $z \in K \backslash\{\zeta\}$. Finally, near $\zeta$, $\operatorname{Re} G(z)=$ $-\varepsilon^{-1} \operatorname{Re} F(z)+\varepsilon^{-2}\left(\operatorname{Im} F^{\prime}\right)^{2}+0\left(|z-\zeta|^{3}\right)$ from which it follows that $G_{\zeta}=$ $2 \varepsilon G$ has the required properties.

If $S$ is a real subspace of $C^{n}$ and $Y$ is any subset we denote by $d_{S}(Y)$ the diameter (in the Euclidean metric) of the (real) orthogonal projection of $Y$ on $S$.

Let $K$ be a compact holomorphically convex subset of $C^{n}$ and suppose $\partial K$ is strictly pseudoconvex in a neighborhood of a point $\zeta \in \partial D$. Then in a neighborhood of $\zeta$ we can write $\partial K=\{z: \rho(z)=0\}$ where $\rho(z)$ is strictly plurisubharmonic in a neighborhood of $\zeta$ and $\operatorname{grad} \rho \neq 0$. The vector $i \operatorname{grad} \rho$ is orthogonal to grad $\rho$ and so lies in the (real) tangent space to $\partial K$ at $\zeta$; let $T(\zeta)$ be the orthogonal complement to $i \operatorname{grad} \rho$ in this space. Then $T(\zeta)$ is the unique complex subspace of the real tangent space with complex dimension $n-1$. Let $L(\zeta)$ be the real 
line spanned by the vector $i \operatorname{grad} \rho$.

If $E$ is any subset of $\partial K$ we denote by $H_{E}^{\infty}$ the set of all bounded continuous functions on $K^{0} \cup E$ which are analytic on $K^{\circ}$. We define $A(K)=H_{\partial K}^{\infty}$.

Theorem 1. Let $F$ be a subset of $\partial K$ such that $\partial K$ is strictly pseudoconvex in a neighborhood of $F$. Suppose that for every $\varepsilon>0$ the set $F$ can be covered by a sequence $\left\{V_{i}\right\}$ of open sets with diameter $<\varepsilon$ such that if $\zeta_{i} \in F \cap V_{i}$ for each $i$ then $\sum_{i} d_{L\left(\zeta_{i}\right)}\left(V_{i}\right)<\varepsilon$ and $\sum_{i}\left\{d_{T\left(\zeta_{i}\right)}\left(V_{i}\right)\right\}^{2}<$ $\varepsilon$. Let $V$ be a neighborhood of $F$, let $\eta>0$, and let $g$ be a bounded continuous function on $F$ with $\|g\| \leqq 1$.

Then we can find $f \in H_{F \cup(K \backslash \bar{F})}^{\infty}$ with $f \mid F=g,\|f\| \leqq 1$, and $|f|<\eta$ on $K \backslash V$.

The proof will be split up into lemmas.

Lemma 2. Let $F, V$ and $\eta$ be as in the theorem. Then we can find $f \in H_{F \cup(K \backslash \bar{F})}^{\infty}$ with $f=1$ on $F,\|f\| \leqq 2$ and $|f|<\eta$ on $K \backslash V$.

Proof. For each $\zeta \in F$ we choose $m_{\zeta}, M_{\zeta}>0$, and a function $G_{\zeta} \in$ $H(K)$ as in Lemma 1.

If $W_{\zeta}$ is a sufficiently small open neighborhood of $\zeta$, then whenever $\zeta \in U \subseteq W_{\zeta}$ and $z \in U \cap \partial K$ we have

$$
\begin{aligned}
& \left|G_{\zeta}(z)\right| \leqq \operatorname{Re} G_{\zeta}(z)+\left|\operatorname{Im} G_{\zeta}(z)\right| \\
& \quad \leqq A_{\zeta}|z-\zeta|^{2}+\left|<\operatorname{grad}\left(\operatorname{Im} G_{\zeta}\right)(\zeta), z-\zeta>\right| \\
& \quad \leqq 2 A_{\zeta}\left(d_{1}^{2}+d_{2}^{2}\right)+|\operatorname{grad} \rho(\zeta)| d_{1} \\
& \quad \leqq B_{\zeta}\left(d_{1}+d_{2}^{2}\right)
\end{aligned}
$$

where $d_{1}=d_{L(\zeta)}(U), d_{2}=d_{T(\zeta)}(U), A_{\zeta}, B_{\zeta}$ do not depend on $z$, and $\langle$, $\rangle$ denotes the real scalar product.

For each positive integer $n$ let

$$
F_{n}=\left\{\zeta \in F: B_{\zeta}<n, \Delta(\zeta, 1 / n) \subseteq W_{\zeta}, m_{\zeta} d(\zeta, K \backslash V)^{2}>1 / n, m_{\zeta}>1 / n\right\} .
$$

Then $F=\bigcup_{n} F_{n}$. For each $n$ we choose a sequence $\left\{V_{i}^{(n)}\right\}$ of open sets with diameter less than $1 / n$ such that each point of $F_{n}$ is contained in infinitely many $V_{i}^{(n)}$, and $\sum_{i}\left\{d_{L\left(\zeta_{i}^{n}\right)}\left(V_{i}^{n}\right)+\left(d_{T\left(\zeta_{i}^{n}\right)}\left(V_{i}^{n}\right)\right)^{2}\right\}<\eta n^{-2} 2^{-n-2}$ for some choice of $\zeta_{i}^{(n)} \in V_{i}^{(n)} \cap F_{n}$. Renumber the collection of all $V_{i}^{(n)}$ as $V_{1}, V_{2}, \cdots$. For each $j$ choose $n_{j}$ so that $V_{j}=V_{i}^{\left(n_{j}\right)}$ for some $i$, and let $\zeta_{j}=\zeta_{i}^{\left(n_{j}\right)}$. Let $G_{j}=G_{\zeta_{j}}$. Writing $c_{j}=d_{L\left(\zeta_{j}\right)}\left(V_{j}\right)+\left\{d_{\left.T_{(\zeta}\right)}\left(V_{j}\right)\right\}^{2}$ we define

$$
B_{r}(z)=\prod_{j=1}^{r} \frac{G_{j}(z)}{2 n_{j} c_{j}+G_{j}(z)}, z \in K, r=1,2, \cdots
$$


Then $B_{r} \in H(K)$ and $\left|B_{r}\right| \leqq 1$ on $K$. We claim that $\left\{B_{r}\right\}$ converges pointwise on $F \cup(K \backslash \bar{F})$ to a limit $B$ which is continuous on $F \cup(K \backslash$ $\bar{F})$, analytic on $K^{0}$, zero at each point of $F$, with $\|B\| \leqq 1$ and $\mid 1-$ $B \mid<\eta$ on $K \backslash V$.

If $z \in K \backslash V$ then $\operatorname{Re} G_{j}(z) \geqq m_{\zeta_{j}}\left|z-\zeta_{j}\right|^{2}>1 / n_{j}$, so

$$
\begin{aligned}
\sum_{j=1}^{\infty}\left|1-\frac{G_{j}(z)}{2 n_{j} c_{j}+G_{j}(z)}\right| & =\sum_{j=1}^{\infty} \frac{2 n_{j} c_{j}}{\left|2 n_{j} c_{j}+G_{j}(z)\right|} \\
& \leqq \sum_{j=1}^{\infty} 2 n_{j}^{2} c_{j}<\eta / 2,
\end{aligned}
$$

which proves that $B_{r}(z)$ converges to a limit $B(z)$ with $|1-B(z)|<\eta$.

If $z \in K \backslash \bar{F}$ then

$$
\sum_{j=J_{0}}^{\infty} \frac{2 n_{j} c_{j}}{\left|2 n_{j} c_{j}+G_{j}(z)\right|} \leqq \sum_{j=j_{0}}^{\infty} \frac{2 n_{j}^{2} c_{j}}{\left|z-\zeta_{j}\right|^{2}} \leqq d\left(z, F^{\prime}\right)^{-2} \sum_{j=\jmath_{0}}^{\infty} 2 n_{j}^{2} c_{j}
$$

The series on the right converges, so $B_{r}$ converges uniformly to a limit $B$ on sets at positive distance from $F$, so $B$ is continuous on $K \backslash \bar{F}$ and analytic on $K^{\circ}$.

Finally let $z \in F$. Then $z \in V_{j}$ for infinitely many $j$. For each such $j$ we have $V_{j} \cong W_{\zeta_{j}}$ and for all $w \in W_{\zeta_{j}}$,

$$
\left|\frac{G_{j}(w)}{2 n_{j} c_{j}+G_{j}(w)}\right| \leqq \frac{n_{j} c_{j}}{2 n_{j} c_{j}}=\frac{1}{2} .
$$

It follows that $B_{r}(z) \rightarrow 0$ and $\lim \left|B_{r}\right|$ is continuous at $z$. Thus $B$ has the asserted properties, and $f=1-B$ satisfies the requirements of the theorem.

Lemma 3. Let $X$ be a compact subset of $K, W$ a neighborhood of $X$, and $h$ a continuous function on $K$ with support in $X$ such that $\|h\| \leqq 1$. Let $\eta>0$.

Then there exists $f \in H_{F \cup(K \backslash \bar{F})}^{\infty}$ such that $|f-h|<\eta$ on $F,\|f\| \leqq$ 3 , and $|f|<\eta$ on $K \backslash W$.

Proof. Choose $0<\delta<d(X, K \backslash W)$ so small that $|h(x)-h(y)|<$ $\eta / 8$ whenever $x, y \in K,|x-y|<\delta$. We can easily find an integer $N>0$, compact sets $X_{1} \cdots X_{r}$ contained in $X$, and open sets $W_{1} \cdots$ $W_{r}$, with diameters $<\delta$, with $W_{i} \supseteqq X_{i}, W_{i} \subseteq W$, such that

(a) if $x \in X$ and $N_{x}$ is the number of integers $i$ in $\{1, \cdots, r\}$ for which $x \in X_{i},\left|N_{x}-N\right|<\eta N / 8$

(b) if $x \in C^{n}$ the number of integers $i$ for which $x \in W_{i} \backslash X_{i}$ is less than $\eta N$.

Let $F_{i}=F \cap X_{i}$. For $i=1,2 \cdots r$ we can find by Lemma 2 functions $f_{i} \in H_{F_{i} \cup\left(K \backslash \bar{F}_{i}\right)}^{\infty}$ with $f_{i}=1$ on $F_{i},\left\|f_{i}\right\| \leqq 2$ and $\left|f_{i}\right|<\eta / 3 r$ on $K \backslash W_{i}$. 
Choose $x_{i} \in X_{i}$ for each $i$ and put $f(z)=1 / N \sum_{i=1}^{r} f_{i}(z) h\left(x_{i}\right), z \in F \cup(K \backslash \bar{F})$. Clearly $f \in H_{F \cup(K \backslash \bar{F})}^{\infty}$ and $\|f\| \leqq 3$ by (a). If $z \in K \backslash W$ then $\left|f_{i}(z)\right|<\eta / r$ for each $i$ so $|f(z)|<\eta$.

Finally let $z \in F$. Then

$$
\begin{aligned}
f(z) & =\frac{1}{N}\left(\sum_{z \in X_{i}}+\sum_{z \in W_{i} \backslash X_{i}}+\sum_{z \notin W_{i}} f_{i}(z) h\left(x_{i}\right)\right. \\
& =f_{1}(z)+f_{2}(z)+f_{3}(z), \text { say. }
\end{aligned}
$$

We have

$$
\begin{aligned}
\mid f_{1}(z) & -h(z)|\leqq| \frac{1}{N} \sum_{z \in X_{i}} f_{i}(z)\left(h(z)-h\left(x_{i}\right)\right) \mid \\
& +\left|1-\frac{N_{z}}{N}\right|<\frac{\eta N_{z}}{8 N}+\left|1-\frac{N_{z}}{N}\right|<\eta / 3,
\end{aligned}
$$

by (a), since $\left|z-x_{i}\right|<\delta$. Moreover, $\left|f_{2}(z)\right|<\sum_{i=1}^{r} \eta / 3$, by (b) and $\left|f_{3}(z)\right|<\sum_{i=1}^{r} \eta / 3 r=\eta / 3$, so that we have $|f(z)-h(z)|<\eta$ as required.

LEMMA 4. With $F$ as in the theorem, if $W$ is any open neighborhood of $F$ and $h$ a bounded continuous function on $W$ with $\|h\| \leqq 1$, we can find $G \in H_{F \cup(K \backslash \bar{F})}$ with $|G-h|<\eta$ on $F,\|G\| \leqq 7$, and $|G|<\eta$ outside $W$.

Proof. Choose a sequence $\left\{W_{n}\right\}$ of relatively compact open subsets of $W$ with $W=\bigcup_{n=1}^{\infty} W_{n}$, such that $\bar{W}_{m} \cap \bar{W}_{n}=\varnothing$ if $|m-n|>1$. We can write $h=\sum_{n=1}^{\infty} h_{n}$ on $W$ where $h_{n} \in C(K)$ has support in $W_{n}$ and $\left\|h_{n}\right\| \leqq 1$. By Lemma 3 for each $n$ we can find $f_{n} \in H_{F \cup(K \backslash \bar{F})}^{\infty}$ with $\left|f_{n}-h_{n}\right|<2^{-n} \eta$ on $F,\left|f_{n}\right|<2^{-n} \eta$ on $K \backslash W$, and $\left\|f_{n}\right\| \leqq 3$. Then $G=$ $\sum_{n=1}^{\infty} f_{n}$ has the required properties.

Proof of Theorem 1. By Lemma 4 and using the fact that $g$ can be approximated uniformly by functions continuous in a neighborhood of $F$, we can construct by induction on $n$ a sequence $\left\{G_{n}\right\}_{n=0}^{\infty}$ in $H_{F \cup(K \backslash \bar{F})}^{\infty}$ such that, writing $f_{n}=G_{0}+\cdots+G_{n}$ we have:

$$
\left|G_{0}-g\right|<\lambda / 7 \text { on } F \text {, }
$$

$$
\left|G_{n}+f_{n-1}-\left(1+\lambda+\cdots+\lambda^{n}\right) g\right|<\frac{\lambda^{n+1}}{7}
$$

on $F, n>1$, where $\lambda=9 / 10$

$$
\begin{gathered}
\left\|G_{n}\right\| \leqq 7\left\|f_{n-1}-\left(1+\lambda+\cdots+\lambda^{n}\right) g\right\|_{F}<8 \lambda^{n} \\
\left\|f_{n}\right\| \leqq 1+\lambda+\cdots+9 \lambda^{n} .
\end{gathered}
$$


(To get $(3)_{n}$ observe that by $(1)_{n-1}$ we have $\left|f_{n-1}\right|<1+\lambda+\cdots+$ $\lambda^{n-1}+\lambda^{n} / 7$ on $F$, and hence on a neighborhood of $F$; if we make $\left|G_{n}\right|<$ $\lambda^{n-1} / 10$ outside this neighborhood then $(3)_{n}$ follows from $(2)_{n}$ and $\left.(3)_{n-1}\right)$.

$$
\left|G_{n}\right|<2^{-n} \text { on } K \backslash V \text {. }
$$

Then $(2)_{n}$ shows that $f_{n} \rightarrow G$ say uniformly on $K$, so $G \in H_{F^{\prime} \cup(K \backslash \bar{F})}^{\infty}$; by $(1)_{n} G=10 g$ on $F$ and by $(3)_{n}\|G\| \leqq 10$. Finally by $(4)_{n}|G|<\eta$ on $K \backslash V$. Then $f=(1 / 10) G$ is the required function.

REMARK. The metric condition on $F$ in Theorem 1 is clearly satisfied if $F$ has zero one-dimensional Hausdorff measure; however it is also satisfied by sets which are thicker in the direction of the complex tangent space, e.g. any smooth arc in $\partial K$ whose tangent at each point lies in the complex tangent space.

If $F$ is compact then of course it is a peak interpolation set, so Theorem 1 extends the Rudin-Carleson theorem. The extension to nonclosed sets in the case of the disc has been obtained independently by Detraz [1], and subsequently generalized to other domains in the plane by A. Stray (private communication).

If we assume that $F$ has zero one-dimensional Hausdorff measure then we can make do with a weaker pseudoconvexity hypothesis at the points of $F$. We say that $\partial K$ is point pseudoconvex at $\zeta$ if there exists a neighborhood $N$ of $\zeta$ and a real $C^{2}$ strictly plurisubharmonic function $\rho$ in $N$ such that $\rho(\zeta)=0$ and $\rho(z) \leqq 0$ in $N \cap K$.

THEOREM 2. Let $K$ be holomorphically convex, and let $F$ be a subset of $\partial K$ with zero one-dimensional Hausdorff outer measure such that $\partial K$ is point pseudoconvex at each point of $F$. Let $V$ be a neighborhood of $F$ in $K$, let $\eta>0$, and let $g$ be a bounded continuous function on $F$ with $\|g\| \leqq 1$.

Then we can find $f \in H_{F \cup(K \backslash \bar{F})}^{\infty}$ with $f \mid F=g,\|f\| \leqq 1$ and $|f|<\eta$ on $K \backslash V$.

Proof. We show that the conclusion of Lemma 2 holds; the rest of the proof is just as before. As in the proof of Lemma 2 for each $\zeta \in F$ we can find positive constants $m_{\zeta}$ and $M_{\zeta}$, a neighborhood $W_{\zeta}$ of $\zeta$, and $G_{\zeta} \in H(K)$ such that

(a) $m_{\zeta}|\zeta-z|^{2} \leqq \operatorname{Re} G_{\zeta}(z), \quad z \in K$

(b) $\left|G_{\zeta}(z)\right| \leqq M_{\zeta}|\zeta-z|, \quad z \in K$.

Then whenever $\zeta \in U \subset W_{\zeta}$ and $z \in U$ we have $\left|G_{\zeta}(z)\right| \leqq M_{\zeta} \operatorname{diam}(U)$. We define $F_{n}$ as before and cover $F_{n}$ by balls $\Delta_{i}^{(n)}$ such that $\sum_{i} \operatorname{diam}\left(\Delta_{i}^{n}\right)<$ $\varepsilon n^{-2} 2^{-n-2}$. The rest of the proof goes just as before, with $c_{j}$ replaced by $\operatorname{diam}\left(\Delta_{j}\right)$. 
COROLLARY. Let $F$ be a compact subset of $\partial K$ with zero 1-dimensional Hausdorff measure and assume $\partial K$ is point pseudoconvex at each point of $F$. Then $F$ is a peak interpolation set for $A(K)$.

Finally we remark that the functions obtained in Theorem 1 and 2 are actually pointwise limits on $K^{0}$ of bounded sequences in $H(K)$; this follows from the construction. If $F$ is compact the peak-interpolating functions constructed are in $\bar{H}(K)$; in this case the proof simplifies somewhat since it is only necessary to take finite products in Lemma 2 and the theorem follows from Lemma 2 by general theorems on peak interpolation sets.

\section{REFERENCES}

1. J. Detraz, Algebres de fonctions analytiques dans le disque, Ann. Sci. École Norm. Sup., 3 (1970), 313-352.

2. T. W. Gamelin, Uniform Algebras, Prentice-Hall, Englewood Cliffs, N. J., 1969.

3. R. C. Guning and H. Rossi, Analytic Functions of Several Complex Variables, Prentice-Hall, Englewood Cliffs, N. J, 1965.

4. L. Hormander, An Introduction to Complex Analysis in Several Variables, Van Nostrand, Princeton, 1967.

Received May 10, 1971. The work of the first author was partially supported by NSF Grant GP-19067 and the work of the second author was partially supported by NAVF, Norway and NSF Grant GP-11475.

University of California, Los ANgeles 



\section{PACIFIC JOURNAL OF MATHEMATICS}

\section{EDITORS}

H. SAMELSON

Stanford University

Stanford, California 94305

C. R. Hовву

University of Washington

Seattle, Washington 98105
J. DugundJI

Department of Mathematics

University of Southern California

Los Angeles, California 90007

RICHARD ARENS

University of California

Los Angeles, California 90024

\section{ASSOCIATE EDITORS}
E. F. BECKENBACH
B. H. NeumanN
F. WOLF
K. YOSHIDA

\section{SUPPORTING INSTITUTIONS}

UNIVERSITY OF BRITISH COLUMBIA

CALIFORNIA INSTITUTE OF TECHNOLOGY

UNIVERSITY OF CALIFORNIA

MONTANA STATE UNIVERSITY

UNIVERSITY OF NEVADA

NEW MEXICO STATE UNIVERSITY

OREGON STATE UNIVERSITY

UNIVERSITY OF OREGON

OSAKA UNIVERSITY
UNIVERSITY OF SOUTHERN CALIFORNIA

STANFORD UNIVERSITY

UNIVERSITY OF TOKYO

UNIVERSITY OF UTAH

WASHINGTON STATE UNIVERSITY

UNIVERSITY OF WASHINGTON

$\stackrel{*}{*} \stackrel{*}{*} \stackrel{*}{*}{ }^{*}$ AMERICAN MATHEMATICAL SOCIETY

NAVAL WEAPONS CENTER 


\section{Pacific Journal of Mathematics}

\section{Vol. 41, No. $1 \quad$ November, 1972}

Anatole Beck and Peter Warren, Weak orthogonality.................

Jonnie Bee Bednar and Howard E. Lacey, Concerning Banach spaces whose duals are abstract $L$-spaces.............................

Louis Harvey Blake, Canonical extensions of measures and the extension of regularity of conditional probabilities..........................

R. A. Brooks, Conditional expectations associated with stochastic processes ..........................................

Theodore Allen Burton and Ronald Calvin Grimmer, On the asymptotic behavior of solutions of $x^{\prime \prime}+a(t) f(x)=e(t) \ldots \ldots \ldots \ldots \ldots \ldots$

Stephen LaVern Campbell, Operator-valued inner functions analytic on the closed disc ............................................

Yuen-Kwok Chan, A constructive study of measure theory...

Alexander Munro Davie and Bernt Karsten Oksendal, Peak interpolation sets for some algebras of analytic functions ...................

H. P. Dikshit, Absolute total-effective $\left(N, p_{n}\right)(c, 1)$ method ...............

Robert E. Edwards, Edwin Hewitt and Kenneth Allen Ross, Lacunarity for

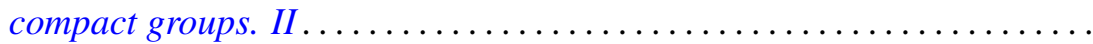

James Daniel Halpern, On a question of Tarski and a maximal theorem of Kurepa

Gerald L. Itzkowitz, A characterization of a class of uniform spaces that admit an invariant integral

Mo Tak Kiang, Semigroups with diminishing orbital diameters ..

Glenn Richard Luecke, A class of operators on Hilbert space ...

R. James Milgram, Group representations and the Adams spectral sequence. . .

G. S. Monk, On the endomorphism ring of an abelian p-group, and of a large subgroup...

Yasutoshi Nomura, Homology of a group extension ...

R. Michael Range, Approximation to bounded holomorphic functions on strictly pseudoconvex domains...

Norman R. Reilly, Inverse semigroups of partial transformations and $\theta$-classes.

Chris Rorres, Strong concentration of the spectra of self-adjoint operators

Saharon Shelah, A combinatorial problem; stability and order for models and theories in infinitary languages.

George Gustave Weill, Vector space decompositions and the abstract

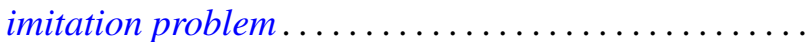

